\title{
ESTIMACIÓN DEL TIEMPO EFECTIVO DE ATENCIÓN EN CONSULTA EXTERNA DEL SERVICIO DE MEDICINA DEL CENTRO DE SALUD LA ESPERANZA, TACNA-2018
}

\author{
ESTIMATION OF THE EFFECTIVE \\ TIME OF HEALTHCARE IN THE \\ OUTPATIENT SERVICE OF \\ MEDICINE AT THE HEALTH CENTER \\ LA ESPERANZA, TACNA-2018
}

\author{
Alex Eduardo Tapia Tenorio \\ 1. Maestrante en Gestión de Servicios de \\ Salud. Médico Asistencial del Centro de \\ Salud La Esperanza Microred Cono Norte \\ Red de Salud Tacna.
}

\section{RESUMEN}

El propósito de esta investigación fue estimar el tiempo efectivo de atención en consulta externa del servicio de medicina en el Centro de Salud La Esperanza en Tacna en el año 2018. La investigación fue de tipo no experimental, descriptiva, cuantitativa, transversal y aplicada. Se tomó una muestra de 373 atenciones para un $p=0.005$ y q $=0.005$ con un nivel de confianza de $95 \%$ por muestreo aleatorio simple. Se estimaron los tiempos en tres dimensiones: tiempo para el diagnóstico (anamnesis, examen físico, diagnóstico y exámenes auxiliares), tiempo para el tratamiento (receta médica, prevención de la salud y promoción de la salud) y tiempo para el llenado de formatos auditables (formatos, cuadernos, padrones y otros). La estimación se realizó mediante una encuesta que fue llenada por cada médico durante la consulta externa; posteriormente, se trasladó la información a una base de datos. El tiempo promedio efectivo de las atenciones arrojó una media igual a 36.0572. La prueba estadística de Kruskal-Wallis determinó que $p<\alpha$, $0.000<0.050, \mathrm{Gl}=2$; por tanto, sí existe diferencia estadística significativa entre el llenado de formatos y las otras dimensiones. Los promedios referentes a los tiempos utilizados fueron variados; en atención del diagnóstico fue de 11.1 minutos; en la atención para el tratamiento, 9.4 minutos y en la atención para llenado de formatos administrativos auditables, 15.6 minutos. Tales cifras sobrepasan a los tiempos promedio encontrados en las fuentes de información; consecuentemente, se debe mejorar los procesos de atención. Se concluye que el llenado de formatos en los diferentes procesos de atención disminuye el tiempo de labor clínica del profesional de la salud.

Palabras clave: Tiempo de atención, tiempo de diagnóstico, tiempo de tratamiento, tiempo para el llenado de formatos auditables, tiempo de anamnesis, tiempo de examen físico.

\section{ABSTRACT}

The purpose of the research was to estimate the effective time of the outpatient service of the medical service at the Health Center La Esperanza in Tacna in 2018. It was a nonexperimental, descriptive, quantitative, cross-sectional and applied research. A sample of 373 attentions was taken for a p $=0.005$ and $q=0.005$ with a confidence level of $95 \%$ for simple random sampling. The times were estimated in three dimensions: time for the diagnosis (anamnesis, physical examination, diagnosis and auxiliary tests), time for the treatment (prescription, health prevention and health promotion) and time for the filling of auditable formats (formats, notebooks, registers and others). The estimate was made by means of a survey that was filled out by each doctor during the external consultation. Subsequently, the information was transferred to a database. The average effective time of the attentions showed an average equal to 36.0572. The Kruskal-Wallis statistical test determined that $p$ $<\alpha, 0.000<0.050, \mathrm{Gl}=2$; therefore, there is a significant statistical difference between the filling of formats and the other dimensions. The averages referring to the times used were varied; in attention to the diagnosis, it was 11.1 minutes; in the care for the treatment, 9.4 minutes and in the attention for filling auditable administrative forms, 15.6 minutes. These figures surpass the average times found in the information sources, so the attention processes must be improved. It is concluded that the filling of formats in the different processes of attention reduces the time of clinical work of the health professional.

Keywords: Estimation of the time of care, time of diagnosis, treatment time, time for filling of auditable formats, time of anamnesis, physical examination time. 


\section{INTRODUCCIÓN}

En la atención de pacientes existen dos tipos de actividades; una específica clínica orientada a determinar el diagnóstico y tratamiento; y otra destinada a registrar las actividades realizadas durante la atención. El profesional de la salud hace denodados esfuerzos por cumplir con ambas por lo que se pretende, en primer momento, estimar los tiempos para que, en segunda instancia, se puedan tomar las acciones necesarias a fin de mejorar la calidad de atención. En las fuentes de información internacionales encontradas sobre el tema de tiempo de consulta, destacamos las consideraciones de la Organización Mundial de la Salud, la cual refiere un promedio de 10 minutos de atención médica por persona ${ }^{(1)}$. Adicionalmente, se encontraron artículos referidos a los tiempos que se utilizan en consultas médicas en diferentes países del mundo. Así, un artículo de revisión bibliográfica concluyó que un promedio de 10 a 15 minutos es tiempo suficiente para realizar una serie de intervenciones médicas ${ }^{(2)}$. Por añadidura, un estudio prospectivo midió los tiempos durante la visita médica en atención primaria. Este estudio fue realizado en Menorca-España con una muestra de 289 atenciones, para el cual se contó con la participación de un médico en uso de un cronometro. El estudio determinó que el tiempo medio para visita médica en atención primaria fue de 7.4 minutos con un rango entre 76 segundos y 25 minutos; además, se sugirió que la fase de escucha activa del paciente debe ampliarse y, por tanto, de la atención del paciente como lo planteamos en nuestro estudio ${ }^{(3)(4)}$.

A continuación delimitaremos conceptos de los términos básicos para este estudio. El primer término es la estimación del tiempo. Según el diccionario de la Real Academia Española estimar es calcular o determinar el valor de algo. Tiempo es la magnitud física que permite ordenar la secuencia de los sucesos, y cuya unidad en el sistema internacional es el segundo ${ }^{(5)}$. El segundo término es el tiempo de atención para el diagnóstico. En este estudio consideramos que es el tiempo que se utiliza para llamar al paciente, saludarlo, así como realizar la anamnesis, el examen físico, el diagnóstico y la solicitud o interpretación de los exámenes auxiliares ${ }^{(6)(7)}$. El tercer término es el tiempo de atención para el tratamiento. Aquí, hemos agrupado el tiempo que se usa para la receta médica con sus indicaciones, las actividades de prevención de la salud y las de promoción de la salud. Por último, tenemos el Tiempo de atención para llenado de formatos y otros. Este tiempo está referido al utilizado para llenar el registro diario de atenciones en consulta externa, el formato único de atención, los padrones de las diferentes estrategias, cuadernos de seguimiento, $y$ software de búsquedas de diagnósticos y otros que no sean de atención directa con el paciente.

\section{MATERIALES Y MÉTODOS}

Este es un estudio no experimental porque no existe una manipulación deliberada de la variable a medir. La variable es solo observada para luego analizar los datos que obtengamos. Es así que medimos el tiempo que se utiliza para las diferentes dimensiones propuestas de la consulta externa y luego analizamos su proporcionalidad. La población estuvo constituida por los pacientes que acudieron al servicio de consulta externa. Se realizó un muestreo aleatorio en base a las atenciones del año anterior, donde se calcularon 373 atenciones. Se aplicó una encuesta, la cual fue llenada por el profesional que realizó el acto médico. Posteriormente, se utilizó una base de datos mediante escalas que permitió observar las de mayor frecuencia, medias, y desviación estándar.

\section{RESULTADOS}

La Figura 1 reúne los indicadores según edad del paciente, donde se puede apreciar que el $44.0 \%$ de los pacientes está entre las edades de 0 a 11 años, el $23.9 \%$ está entre las edades de 30 a 59 años. Según sexo del paciente, el $59.5 \%$ es del sexo femenino y el $40.5 \%$ es del sexo masculino.

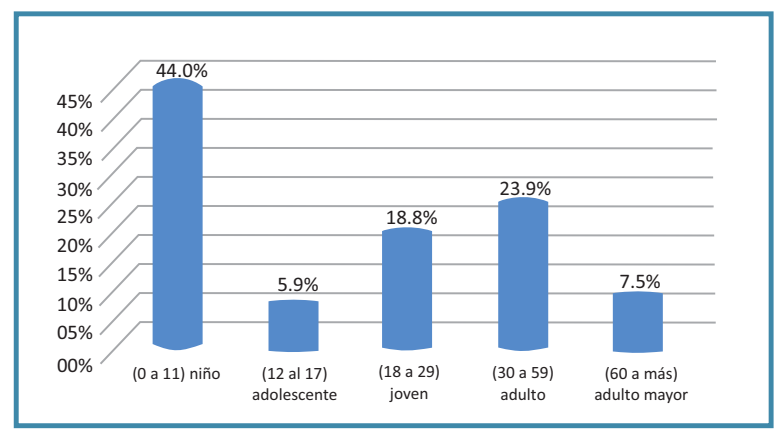

Figura 1. Estimación del tiempo efectivo de atención en consulta externa del servicio de medicina del Centro de Salud la Esperanza, según edad del paciente.

Fuente: Elaboración propia.

En la Tabla 2, observamos rangos de tiempo estimados en diversas actividades de la atención para el diagnóstico. Allí se puede apreciar que las frecuencias 
con mayores porcentajes son: $35.9 \%$ en el rango 1.6 a 3 minutos para la anamnesis; $49.1 \%$ en el rango 2.6 a 4.1 minutos para el examen físico; $42.6 \%$ en el rango 0.25 a 1.26 minutos para el diagnóstico; $46.4 \%$ en el rango 1.5 a 2.4 minutos para los exámenes auxiliares.

Tabla 1. Estimación del tiempo efectivo de atención en consulta externa del servicio de medicina del Centro de Salud La Esperanza, según atención para diagnóstico

\section{ATENCIÓN PARA DIAGNÓSTICO}

\section{TIEMPO DE ANAMNESIS}

1.00 a 1.5 Minutos

1.6 a 3.0 Minutos

3.1 a 4.5 Minutos

4.6 a 6.0 Minutos

6.1 a Mas

\section{TIEMPO DE EXAMEN FÍSICO}

\begin{tabular}{|c|c|c|c|}
\hline 0.9 & a 2.5 Minutos & 114 & $30.6 \%$ \\
\hline 2.6 & a 4.1 Minutos & 183 & $49.1 \%$ \\
\hline 4.2 & 5.7 Minutos & 50 & $13.4 \%$ \\
\hline 5.8 & a 7.3 Minutos & 21 & $5.6 \%$ \\
\hline 7.4 & a Mas & 5 & $1.3 \%$ \\
\hline \multicolumn{4}{|c|}{ TIEMPO DE DIAGNOSTICO } \\
\hline 0.25 & a 1.26 Minutos & 159 & $42.6 \%$ \\
\hline 1.27 & a 2.26 Minutos & 103 & $27.6 \%$ \\
\hline 2.27 & a 3.26 Minutos & 66 & $17.7 \%$ \\
\hline 3.27 & a 4.26 Minutos & 28 & $7.5 \%$ \\
\hline 4.27 & a Más & 17 & $4.6 \%$ \\
\hline \multicolumn{4}{|c|}{ TIEMPO DE EXAMENES AUXILIARES } \\
\hline 0.50 & a 1.4 Minutos & 90 & $24.1 \%$ \\
\hline $1.5 \mathrm{a}$ & 2.4 Minutos & 173 & $46.4 \%$ \\
\hline & a 3.4 Minutos & 93 & $24.9 \%$ \\
\hline $3.5 \mathrm{a}$ & a 4.4 Minutos & 15 & $4.0 \%$ \\
\hline $4.5 \mathrm{a}$ & a Más & 2 & $0.5 \%$ \\
\hline
\end{tabular}

En el Tabla 2 observamos rangos de tiempo estimados con mayores porcentajes de frecuencia. El tiempo utilizado con mayor frecuencia (45.6\%) para la prescripción de la receta médica fue de 1 a 2.4 minutos. Para la prevención de la salud, el rango de tiempo con mayor frecuencia (64.4\%) fue de 1 a 3.8 minutos, uniendo las dos primeras categorías. Para la promoción de la salud, el rango de tiempo con mayor frecuencia (39.1\%) fue de 3.9 a 5.2 .

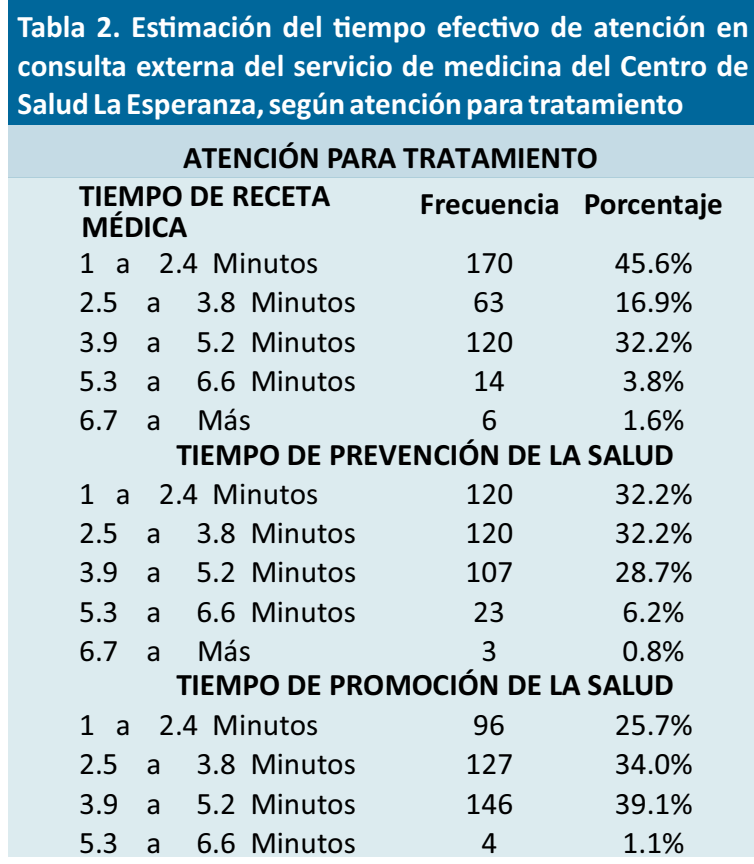

Tabla 3. Estimación del tiempo efectivo de atención en consulta externa del servicio de medicina del Centro de Salud La Esperanza, según atención para llenado de formatos administrativos auditables.

\begin{tabular}{|c|c|c|}
\hline \multicolumn{3}{|c|}{ Tiempo de Llenado de Formato FUA } \\
\hline 1 a 2.4 Minutos & 11 & $2.9 \%$ \\
\hline 2.5 a 3.8 Minutos & 61 & $16.4 \%$ \\
\hline 3.9 a 5.2 Minutos & 189 & $50.7 \%$ \\
\hline 5.3 a 6.6 Minutos & 56 & $15.0 \%$ \\
\hline 6.7 a Más & 56 & $15.0 \%$ \\
\hline \multicolumn{3}{|c|}{ TIEMPO DELLENADO DE FORMATO HISS } \\
\hline 1 a 2.4 Minutos & 94 & $25.2 \%$ \\
\hline 2.5 a 3.8 Minutos & 138 & $37.0 \%$ \\
\hline 3.9 a 5.2 Minutos & 137 & $36.7 \%$ \\
\hline 5.3 a 6.6 Minutos & 3 & $0.8 \%$ \\
\hline 6.7 a Más & 1 & $0.3 \%$ \\
\hline \multicolumn{3}{|c|}{ TIEMPO DE LLENADO DE PADRONES } \\
\hline 1 a 2.4 Minutos & 206 & $55.2 \%$ \\
\hline 2.5 a 3.8 Minutos & 78 & $20.9 \%$ \\
\hline 3.9 a 5.2 Minutos & 89 & $23.9 \%$ \\
\hline \multicolumn{3}{|c|}{ TIEMPO DE LLENADO DE CUADERNOS } \\
\hline 1 a 2.4 Minutos & 137 & $36.7 \%$ \\
\hline 2.5 a 3.8 Minutos & 144 & $38.6 \%$ \\
\hline 3.9 a 5.2 Minutos & 91 & $24.4 \%$ \\
\hline 5.3 a 6.6 Minutos & 1 & $0,3 \%$ \\
\hline \multicolumn{3}{|c|}{ TIEMPO DE LLENADO DE SOFTWARE Y OTROS } \\
\hline 0.4 a 1.4 Minutos & 138 & $37.0 \%$ \\
\hline 1.5 a 2.4 Minutos & 115 & $30.8 \%$ \\
\hline 2.5 a 3.4 Minutos & 41 & $11.0 \%$ \\
\hline 3.5 a 4.4 Minutos & 23 & $6.2 \%$ \\
\hline 4.5 a Más & 56 & $15.0 \%$ \\
\hline
\end{tabular}


En el Tabla 3, podemos observar los rangos de tiempo con mayores frecuencias para el llenado de diversos formatos administrativos auditables. Respecto al tiempo utilizado para el llenado del formato único de atención del asegurado, la mayor frecuencia 50.7\% recayó en el rango 3.9 a 5.2 minutos. En cuanto al llenado del formato de registro diario de actividades en consulta externa la mayor frecuencia con $37 \%$ estuvo en el rango de 2.5 a 3.8 minutos. Por otro lado, la mayor frecuencia (55.2\%) para el llenado de padrones estuvo en el rango de 1 a 2.4 minutos. Seguidamente, el rango 2.5 a 3.8 fue el más frecuente (38.6\%) para el llenado de cuadernos. Finalmente, El llenado de software y otros tuvo mayor frecuencia (37\%) en el rango 0.4 a 1.4 minutos.

\section{CONCLUSIONES}

El tiempo efectivo de atención en consultorio externo de medicina del Centro de Salud La Esperanza, según nuestro estudio, tuvo una media de 36.1 minutos mayor a los 15 minutos que se plantea en la bibliografía revisada. En cuanto a las tres dimensiones estudiadas; se observó que el tiempo para el diagnóstico tuvo un promedio de 11.1 minutos, el tiempo para el tratamiento presentó un promedio de 9.4 minutos y el tiempo de llenado de formatos auditables, de $15.6 \mathrm{~min}$. La prueba estadística de Kruskal-Wallis determinó que $\mathrm{p}<\alpha, 0.000<0.050, \mathrm{Gl}=2$; por tanto, sí existe diferencia estadística significativa entre la atención al paciente y el llenado de formatos auditables.

\section{REFERENCIAS BIBLIOGRÁFICA}

1. Urquiza, R., (2017).Tiempo de consulta, gestión clínica y trabajo diario de un médico de Familia. Revista Médica La Paz, 23(2), 79-83. Recuperado de (2018, 20 de mayo). http://www.scielo.org.bo/scielo.php?script=sci_arttext\&pid=S 1726-89582017000200018

2. Outomuro, D., Actis, A. M., (2013). Estimación del tiempo de consulta ambulatoria en clínica médica. Rev. Med Chile, Vol.141, $361-366$ R e c u p e r a d o d e https://scielo.conicyt.cl/scielo.php?script=sci_arttext\&pid=S00 34-98872013000300012\&lng=es.

3. Seguí,M., Linares, L.,Blanco, W., et al (2004). Tiempos durante la visita médica en atención primaria. Elsevier, 33(2), 496-502. Recuperado de (2018, 20 de mayo) http://www.elservier.es
4. Altisent R.,( 2008, enero) Tiempos mínimos en las consultas médicas. Organización médica colegial. Recuperado de: (2018, 19 d e $m$ a y o ). https://www.cgcom.es/sites/default/files/08_01_25_tiempo_ consulta_0.pdf

5. Asociación de Academias de lengua española. (2014). Diccionario de la lengua española Recuperado de http://www.rae.es/diccionario-de-la-lengua-espanola/la-23aedicion-2014.

6. Swartz, M., (2015). Tratado de semiología anamnesis y exploración física. 6. ed Barcelona, España: Elsevier, Recuperado de (2018, 20 de mayo). http://dl.free.fr/sXneW8eBd

7. Cardelach, F., Grau, J. y Rozman, C., (2014) Compendio de anamnesis y exploración física. Barcelona, España: Elsevier;. Recuperado de (2018, 20 de mayo). http://dl.free.fr/hvxtdkU1s

\section{Correspondencia}

Alex Eduardo Tapia Tenorio

E-mail: med.alextapia15@hotmail.com
Fecha de recepción: 20 de octubre de 2018 Fecha de aceptación: 25 de febrero de 2019 